In Cres. Vol. $4 N^{\circ}$ 2: pp. 245-256, 2013

\title{
LA EDUCACIÓN RELIGIOSA Y SU INFLUENCIA EN LA FORMACIÓN DE VALORES EN LOS ESTUDIANTES DE EDUCACIÓN SECUNDARIA*
}

\author{
RELIGIOUS EDUCATION AND ITS INFLUENCE \\ ON FORMATION OF VALUES OF \\ HIGH SCHOOL STUDENTS
}

Ana Isabel Arellano Carranza ${ }^{l}$

\section{RESUMEN}

La investigación tuvo como objetivo general determinar el nivel de influencia de la educación religiosa católica en la formación de valores en los estudiantes del segundo grado de educación secundaria 2010, de la institución educativa "San Jacinto", distrito de Nepeña. La metodología de la presente investigación es cuantitativa, dado que el propósito fue describir la actuación en términos cuantificables de dos determinadas variables. El diseño de la investigación fue el descriptivo correlacional, en el cual la investigadora buscaba establecer las relaciones entre la formación religiosa que reciben los estudiantes del nivel educativo secundario y la formación en valores que estos poseen. La población de estudio estuvo integrada por 100 estudiantes del segundo grado de educación secundaria de la I.E. San Jacinto del valle de Nepeña. Dada la naturaleza de la investigación, se utilizó como técnica la encuesta, aplicándose a los estudiantes cuestionarios que permitieron recabar datos sobre la actuación de las dos variables de investigación: la educación religiosa y la formación en valores.

De los resultados obtenidos se observa que la variable educación religiosa católica tiene una correlación positiva alta con la variable Formación en valores, alcanzando un valor promedio de 0,71189 en la escala de Pearson. Por ello, al concluir la investigación, se puede afirmar que existe una influencia significativa (correlación positiva alta) de la educación religiosa católica en la formación en valores en los estudiantes del segundo grado de educación secundaria 2010 de la I.E. "San Jacinto", distrito de Nepeña.

PALABRAS CLAVE: Educación religiosa católica, Pastoral educativa y Formación de valores.

* Recibido: 15 de mayo del 2013; aprobado: 14 de octubre del 2013.

1 Magister en Educación. Docente de la Escuela Profesional de Ciencias Religiosas de la Universidad Católica Los Ángeles de Chimbote. 


\section{ABSTRACT}

This investigation aimed at determining the level of influence of the Catholic Religious Education in the formation of values in second grade students of secondary school 2010 of San Jacinto's educational institution, Nepeña District.

The methodology used in this research is quantitative, since the purpose was to describe the action in measurable terms of two specific variables. The research design was correlational descriptive; on which the researcher seeks to establish the relationships between catholic religious formation which the students receive in secondary school and the formation of values that they possess. The population under study consisted of 100 students of the second grade of secondary school from San Jacinto's educational institution in Nepeña's valley. Due to the nature of the research, the technique used was the survey. Questionnaires were applied to the students, which allowed to collect data about the performance of the two research variables such as catholic religious education and values formation.

The results obtained in this study show that the variable "Catholic Religious Education" has a positive high correlation with the variable "Values formation" reaching an average value of 0,71189 in the Pearson scale. Therefore, after the investigation, it can be stated that there is a significant influence (positive high correlation) of the Catholic Religious Education in formation of values of the second grade students of secondary school of 2010 IE "San Jacinto", Nepeña's District.

KEY WORDS: Catholic religious education, pastoral education and formation of values.

\section{INTRODUCCIÓN}

Hoy, que la sociedad en general, afronta una diversidad de problemas, los valores han dejado de ser parte importante en la vida de muchas personas, principalmente aquellos que se relacionan con la convivencia humana, por lo cual es muy necesario rescatar una auténtica práctica de valores que han de orientar las acciones en beneficio de una sociedad más justa y humana.

Se observa cómo los problemas sociales, la delincuencia, desintegración familiar, alcoholismo, prostitución y pandillaje, entre otros, ocasionan serios estragos en la comunidad, no solo a nivel personal sino también a nivel familiar y social. Esto se puede constatar en el contacto diario con las personas, especialmente con los jóvenes de las instituciones educativas. Conscientes de que la educación es el principal instrumento a través del cual se procura formar personas idóneas para la convivencia adecuada dentro de la sociedad, es importante la formación y vivencia de valores dentro del ambiente familiar, ya que de él dependerán las actitudes y conductas que muestren las personas en los diferentes ámbitos en los que se desenvuelva. 
En este proceso formativo la educación cumple un papel importante, ya que no consiste sólo en instruir, transmitir conocimientos, sino también en formar y practicar la vivencia de valores; esto se ve reflejado en los programas curriculares que orientan la formación educativa formal en sus diferentes niveles: inicial, primaria, secundaria y superior, los mismos que intentan responder a la crisis de valores por la que se atraviesa.

Los obispos del Perú, en su carta pastoral "Caminemos con el Señor de la Historia, de la Vida y de la Esperanza", ${ }^{1}$ recuerdan que tras la crisis socioeconómica y política que experimenta el país existe también una crisis religiosa, en la que se han trastocado los valores fundamentales de la vida y de la convivencia social en el país.

En este sentido, se puede deducir que el problema de fondo es la crisis moral y religiosa de la mayoría de responsables del bienestar del país. Entre ellos, los padres de familia, los docentes, las autoridades civiles, militares y religiosas. Pero también los medios de comunicación son responsables, pues no permiten realmente dar una buena y adecuada educación.

Es importante especificar que la realidad del deterioro moral se va generalizando en todos los ámbitos, especialmente el familiar, resquebrajando las relaciones entre padres e hijos, entre hermanos y parientes, entre las autoridades y subordinados en general, donde el respeto por la dignidad de la persona va perdiendo su significado, prevaleciendo la indiferencia, la ingratitud, el egoísmo y la avaricia. Un ejemplo claro de esta situación, son las miles de madres solteras y cientos de miles de niños viviendo en situaciones paupérrimas, material y moralmente.

Hoy los padres abandonan a sus hijos o los dejan a cargo de familiares, buscando oportunidades laborales. Muchos de estos jóvenes crecen solos, sin la formación adecuada que debe recibirse en la familia, primera escuela de virtudes, tal como lo plantea la iglesia en la Constitución Conciliar Gaudium et spes².

La problemática descrita es la que vive el Perú tanto a nivel nacional como regional, habiéndose agudizado últimamente la violencia familiar, el alcoholismo, la drogadicción, el pandillaje y la delincuencia. En el ámbito local, no se está ajenos de estas realidades, sino basta ver los noticieros o leer en los diarios locales, principalmente el diario "Mar Adentro", que en varios de sus artículos publicados mensualmente hace referencia a diversos problemas que atraviesan los jóvenes como consecuencia del abandono que viven en sus familias y al deterioro de los valores morales en su entorno. Estos artículos refieren, sobre todo, 
el nuevo juego en nuestros adolescentes como las "peras malogradas", o los embarazos adolescentes ${ }^{3}$, los cuales se evidencias en las instituciones educativas, o también el alcoholismo ${ }^{4}$, o el famoso bullying, que muchas veces comienza en el hogar ${ }^{5}$ y se repite en las II.EE. y que casi siempre termina en suicidio ${ }^{6}$.

Estas situaciones no son ajenas a la realidad que le toca vivir a la comunidad de San Jacinto, ubicada en el valle de Nepeña, al sur de la provincia Del Santa. En esta comunidad se ubica la I. E. del mismo nombre, que alberga a estudiantes que vienen de los diferentes anexos que conforman el valle; muchos de ellos proceden de familias disfuncionales, originadas por la separación de los padres, la emigración en búsqueda de oportunidades laborales, jornadas laborales extensas, duplicidad de compromisos familiares asumidos especialmente por los papás. Esto genera conflictos en el desarrollo de los estudiantes y lo podemos evidenciar en la baja autoestima, el uso del vocabulario inadecuado y agresivo, la irresponsabilidad en el cumplimiento de sus tareas, la indiferencia en los momentos de oración y la poca reflexión en el área de educación religiosa.

La búsqueda de respuestas a esta preocupación es la que ha conllevado a realizar un estudio serio de la realidad y una reflexión sobre sus resultados, con el fin de contribuir en la formación axiológica cristiana de los jóvenes.

\section{PROBLEMA}

¿Qué influencia significativa tuvo la educación religiosa católica en la formación de valores en los estudiantes del segundo grado de educación secundaria 2010, de la Institución Educativa "San Jacinto", del distrito de Nepeña?

\section{OBJETIVO GENERAL}

Determinar el nivel de influencia de la educación religiosa católica en la formación de valores en los estudiantes del segundo grado de educación secundaria 2010, de la Institución Educativa "San Jacinto", del distrito de Nepeña.

\section{OBJETIVOS ESPECÍFICOS}

a. Identificar los valores predominantes en los estudiantes del segundo grado de educación secundaria 2010 de la Institución Educativa "San Jacinto". 
b. Identificar el nivel de correlación entre la Pastoral Educativa y formación de valores en los estudiantes del segundo grado de educación secundaria 2010 de la Institución Educativa "San Jacinto".

c. Identificar el nivel de correlación entre el área de educación religiosa católica y la formación de valores en los estudiantes del segundo grado de educación secundaria 2010 de la Institución Educativa "San Jacinto".

d. Identificar el nivel de correlación entre la formación familiar y la formación de valores en los estudiantes del segundo grado de educación secundaria 2010 de la Institución Educativa "San Jacinto".

e. Establecer la correlación entre las variables educación religiosa católica y la formación de valores en los estudiantes del segundo grado de educación secundaria de la Institución Educativa "San Jacinto".

\section{MATERIAL Y MÉTODOS}

Esta investigación se ha realizado bajo el enfoque cuantitativo. Se ha seguido un diseño descriptivo correlacional en cuanto busca establecer las relaciones entre la formación religiosa que reciben los estudiantes del nivel educativo secundario y la formación en valores que estos poseen.

La población estuvo integrada por los estudiantes del segundo grado de educación secundaria de la I.E. "San Jacinto" del valle de Nepeña. Se empleó la técnica de la encuesta, que fue escogida en tanto la investigación se ubica en las ciencias sociales y por ser de un manejo sencillo para los estudiantes de educación secundaria.

En función a esta técnica se aplicaron los siguientes instrumentos: Cuestionario sobre educación religiosa católica y cuestionario sobre formación de valores. Estos instrumentos fueron elaborados bajo la modalidad escala de Likert.

\section{RESULTADOS}

Para la presentación de los resultados se utilizó la matriz de ítemes sobre la variable educación religiosa católica con sus tres dimensiones (pastoral educativa, desarrollo del área de educación religiosa y formación familiar) como variable independiente, así como la matriz de ítemes sobre la variable formación en valores (variable dependiente) con sus cinco dimensiones (respeto, puntualidad, responsabilidad, solidaridad y participación). 
Tabla 01

PREDOMINIO DE VALORES PRACTICADOS POR LOS ESTUDIANTES DEL SEGUNDO GRADO DE EDUCACIÓN SECUNDARIA DE LA IE SAN JACINTO

\begin{tabular}{lcccccc}
\hline Valores & $\begin{array}{c}\mathbf{N}^{\circ} \mathbf{d e} \\
\text { ítems } \\
\text { por } \\
\text { valor }\end{array}$ & $\begin{array}{c}\text { Valor } \\
\text { máximo } \\
\text { de cada } \\
\text { ítem }\end{array}$ & $\begin{array}{c}\mathbf{N}^{\circ} \\
\text { total } \\
\text { de } \\
\text { alumnos }\end{array}$ & $\begin{array}{c}\text { Puntaje } \\
\text { máximo } \\
\text { referencial }\end{array}$ & $\begin{array}{c}\text { Puntaje } \\
\text { acumu- } \\
\text { lado }\end{array}$ & $\begin{array}{c}\% \text { del } \\
\text { puntaje } \\
\text { acumu- } \\
\text { lado }\end{array}$ \\
\hline Respeto & 7 & 5 & 100 & 3500 & 3011 & $86,03 \%$ \\
Puntualidad & 6 & 5 & 100 & 3000 & 2511 & $83,70 \%$ \\
Responsabilidad & 8 & 5 & 100 & 4000 & 3357 & $83,93 \%$ \\
Solidaridad & 10 & 5 & 100 & 5000 & 4111 & $82,22 \%$ \\
Participación & 6 & 5 & 100 & 3000 & 2499 & $83,30 \%$ \\
Total & 37 & & & 18500 & 15489 & $83,72 \%$ \\
\hline
\end{tabular}

Fuente: Cuestionario acerca de Formación de Valores.

Consolidado de datos alcanzados por cada valor.

En la tabla 01, sobre el predominio de valores en los estudiantes del segundo grado de educación secundaria de la I.E. "San Jacinto", se observa que el valor que sobresale es el de respeto, obteniendo un $86,03 \%$, seguido de los valores de responsabilidad y puntualidad, que alcanzaron los porcentajes del $83,93 \%$ y $83,70 \%$, respectivamente. Finalmente, están los valores de participación $(83,30 \%)$ y solidaridad $(82,22 \%)$.

El gráfico 1 muestra los datos presentados anteriormente en un diagrama de barras bidimensional que facilita la comparación de los porcentajes obtenidos para cada valor en los sujetos de la muestra.

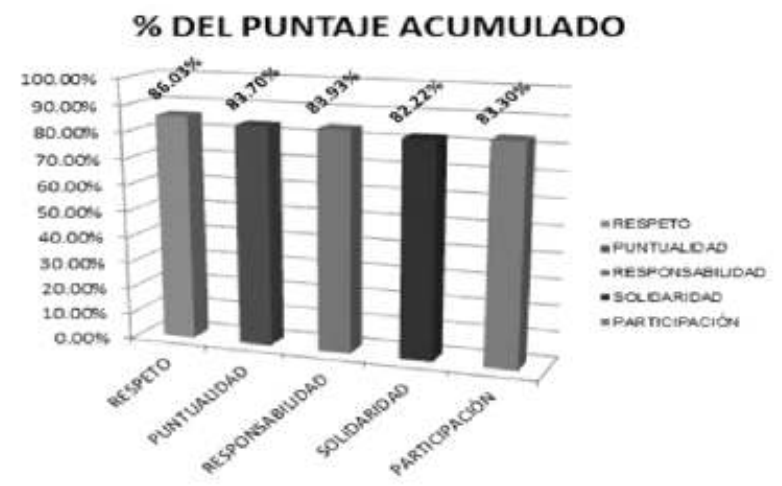

Gráfico 1. Predominio de valores practicados por los estudiantes de la I.E “San Jacinto". Fuente: Tabla 01. 
De la tabla 01 y gráfico 1 presentados se determina la tendencia que la mayoría de los estudiantes ha destacado el valor de respeto como el más predominante.

\section{Tabla 02}

CORRELACIÓN ENTRE LA PASTORAL EDUCATIVA Y LA FORMACIÓN DE VALORES ENTRE LOS ESTUDIANTES DEL SEGUNDO GRADO DE EDUCACIÓN SECUNDARIA DE LA IE DE SAN JACINTO

\begin{tabular}{cccccc}
\hline & $\sum \mathbf{X}$ & $\sum \mathbf{Y}$ & $\sum \mathbf{X Y}$ & $\sum \mathbf{X}^{2}$ & $\sum \mathbf{Y}^{2}$ \\
\hline Valor & 4254 & 15489 & 664373 & 184652 & 2432291 \\
\hline \multicolumn{5}{c}{ Número de sujetos de la muestra $=100$} \\
\hline \multicolumn{5}{c}{$\mathrm{r}=0.49450$} \\
\hline
\end{tabular}

Fuente: Cuestionario acerca de Educación Religiosa Católica.

Consolidado de datos-Correlación Pastoral Educativa y Formación en Valores

En la tabla 02, entre la dimensión pastoral educativa y la variable formación de valores, se ha obtenido un grado de correlación positiva de 0.49450 , la misma que se interpreta como una correlación "media o moderada".

Tabla 03

CORRELACIÓN ENTRE EL ÁREA DE EDUCACIÓN RELIGIOSA

Y LA FORMACIÓN DE VALORES DE LOS ESTUDIANTES

DEL SEGUNDO GRADO DE EDUCACIÓN SECUNDARIA

DE LA IE DE SAN JACINTO

\begin{tabular}{cccccc}
\hline & $\sum \mathbf{X}$ & $\sum \mathbf{Y}$ & $\sum \mathbf{X Y}$ & $\sum \mathbf{X}^{2}$ & $\sum \mathbf{Y}^{2}$ \\
\hline Valor & 6289 & 15489 & 981447 & 401779 & 2432291 \\
\hline
\end{tabular}

Número de sujetos de la muestra $=100$

$\mathrm{r}=0.50925$

Fuente: Cuestionario acerca de Educación Religiosa Católica.

Consolidado de datos-Correlación Formación Religiosa y Formación en Valores.

En la tabla 03, entre la dimensión área de educación religiosa y la variable formación de valores, se ha obtenido un grado de correlación positiva de 0.50925 , la misma que se interpreta como una correlación "media $o$ moderada". 
Tabla 04

CORRELACIÓN ENTRE LA FORMACIÓN FAMILIAR Y

FORMACIÓN DE VALORES EN LOS ESTUDIANTES DEL

SEGUNDO GRADO DE EDUCACIÓN SECUNDARIA

DE LA IE DE SAN JACINTO

\begin{tabular}{cccccc}
\hline & $\sum \mathbf{X}$ & $\sum \mathbf{Y}$ & $\sum \mathbf{X Y}$ & $\sum \mathbf{X}^{2}$ & $\sum \mathbf{Y}^{2}$ \\
\hline Valor & 5712 & 15489 & 896535 & 335492 & 2432291 \\
\hline \multicolumn{5}{c}{ Número de sujetos de la muestra $=100$} \\
\hline \multicolumn{5}{c}{$\mathrm{r}=0.67455$} \\
\hline
\end{tabular}

Fuente: Cuestionario acerca de Educación Religiosa Católica.

Consolidado de datos-Correlación formación familiar y Formación en Valores.

En la tabla 04, entre la dimensión formación familiar y la variable formación de valores, se ha obtenido un grado de correlación positiva de 0.67455 , la misma que se interpreta como una correlación "alta".

Tabla 05

CORRELACIÓN ENTRE LAS VARIABLES EDUCACIÓN RELIGIOSA CATÓLICA Y LA FORMACIÓN DE VALORES PRACTICADOS POR LOS ESTUDIANTES DEL SEGUNDO GRADO DE EDUCACIÓN SECUNDARIA DE LA IE SAN JACINTO

\begin{tabular}{cccccc}
\hline & $\sum \mathbf{X}$ & $\sum \mathbf{Y}$ & $\sum \mathbf{X Y}$ & $\sum \mathbf{X}^{2}$ & $\sum \mathbf{Y}^{2}$ \\
\hline Valor & 16277 & 15489 & 2546255 & 2686883 & 2432291 \\
\hline \multicolumn{5}{c}{ Número de sujetos de la muestra $=100$} \\
\hline \multicolumn{5}{c}{$\mathrm{r}=0.71189$} \\
\hline
\end{tabular}

Fuente: Cuestionario acerca de Educación Religiosa Católica. Consolidado de datos-Correlación Educación Religiosa Católica y Formación en Valores.

En la tabla 05, entre la dimensión educación religiosa católica y la variable formación de valores, se ha obtenido un grado de correlación positiva de 0.71189 , la misma que se interpreta como una correlación "alta".

Asimismo se muestra la tabla 06, referente a la matriz de correlación de las dimensiones presentadas anteriormente con respecto a la formación en valores; siendo $\mathrm{r}_{1}$ el índice de correlación entre pastoral educativa y formación en valores, $r_{2}$ el índice de correlación entre formación religiosa y formación en 
valores y $\mathrm{r}_{3}$ el índice de correlación entre formación familiar y formación en valores.

Tabla 06

MATRIZ DE CORRELACIÓN ENTRE LAS VARIABLES EDUCACIÓN

RELIGIOSA CATÓLICA Y FORMACIÓN EN VALORES

\begin{tabular}{|c|c|c|c|c|}
\hline & $\begin{array}{l}\text { Índice de } \\
\text { correlación } \\
\text { Pastoral } \\
\text { educativa } \\
\text {-formación } \\
\text { en valores }\end{array}$ & $\begin{array}{c}\text { Índice de } \\
\text { correlación Área } \\
\text { de educación } \\
\text { Religiosa } \\
\text {-formación } \\
\text { en valores }\end{array}$ & $\begin{array}{l}\text { Índice de } \\
\text { correlación } \\
\text { Entorno } \\
\text { Familiar } \\
\text {-formación } \\
\text { en valores }\end{array}$ & $\begin{array}{c}\text { Correlación } \\
\text { global entre } \\
\text { educación } \\
\text { religiosa } \\
\text { católica y } \\
\text { formación } \\
\text { en valores }\end{array}$ \\
\hline & $\mathbf{r}^{1}$ & $\mathbf{r}^{2}$ & $\mathbf{r}^{3}$ & $\mathbf{r}$ \\
\hline $\begin{array}{l}\text { Valor de la } \\
\text { correlación }\end{array}$ & 0.49450 & 0.50925 & 0.67455 & 0.71189 \\
\hline \multicolumn{5}{|c|}{ Número de sujetos de la muestra $=100$} \\
\hline
\end{tabular}

Fuente: Cuestionario acerca de Educación Religiosa Católica.

Cuestionario acerca de Formación de Valores.

Se observa que los datos mostrados anteriormente presentan índices de correlación moderados (en el caso de la pastoral educativa y el área de educación religiosa con respecto a la formación en valores) y altos (en el caso de la formación familiar con respecto a la formación en valores). Sin embargo, el índice de correlación general, como lo vimos anteriormente en el Cuadro 6, es un índice alto $(0,71)$.

\section{DISCUSIÓN}

En cuanto al predominio de los valores en los estudiantes del segundo grado de secundaria de la I.E. "San Jacinto", se observa que los cinco valores (respeto, puntualidad, responsabilidad, solidaridad y participación) arrojan porcentajes muy similares. Si bien es cierto, que el valor respeto tuvo el más alto porcentaje acumulado, no se distancia del resto de valores propuestos. El respeto es un valor que en la I.E. "San Jacinto" se ha venido trabajando en diversas áreas curriculares, más allá del Área de Educación Religiosa propiamente dicha; de allí el porcentaje mayor que acumuló. Estos resultados también permiten inferir que los jóvenes estudiantes practican los valores de manera paralela. 
Por otro lado, los valores de participación y solidaridad están ligados directamente a la entrega y compromiso que deberían fomentar los jóvenes en la institución educativa y en la sociedad. Justamente estos valores son los que arrojaron los menores porcentajes de puntaje acumulado, lo cual podría ser reflejo de la tendencia individualista que muestran los jóvenes y el poco compromiso en el desarrollo de su entorno.

En cuanto al nivel de correlación entre la dimensión pastoral educativa y la variable formación en valores, que arroja un nivel medio o moderado, podríamos atribuirlo al poco apoyo que recibe el docente del Área de Educación Religiosa en la I.E. "San Jacinto" por parte de la plana docente, administrativa y padres de familia, con las actividades programadas con los alumnos.

En referencia al nivel de correlación entre la dimensión área de educación religiosa y la variable formación en valores, mostraron un nivel de correlación medio o moderado entre los jóvenes del segundo grado; pero, mayor a la correlación entre pastoral educativa y formación en valores. Esto se debe posiblemente a la formación recibida en el Área de Educación Religiosa, pero sobretodo, al papel fundamental que juega "lo religioso" en la vivencia interior de los jóvenes sanjacinteños, quienes, a pesar de las limitaciones que a todo nivel presentan, siguen conservando su espíritu religioso heredado de sus padres y de la sociedad rural circundante, un pueblo con fervor religioso y con la presencia de lo trascendente actuando en su vida diaria.

La dimensión formación familiar presentó un índice de correlación alta con respecto a la variable formación en valores, a pesar del escaso acompañamiento que experimentan los jóvenes por parte de sus familiares cercanos. En el transcurso de la investigación se observó el gran número de estudiantes provenientes de familias desintegradas, viviendo en estado de abandono o criados por terceras personas, dada la migración familiar o ausencia de los progenitores. Este crítico panorama familiar se convierte en un gran reto para el Área de Educación Religiosa, tanto así que influyen de manera significativa para consolidar una imagen de familia positiva dadas las condiciones que experimentan dichos jóvenes.

Finalmente, se evidenció un alto índice de correlación entre la variable educación religiosa católica y la formación en valores. Este análisis final engloba los datos arrojados en las tres dimensiones correspondientes (pastoral educativa, formación religiosa y formación familiar). Por ello, se puede deducir que, 
en líneas generales, tomando en cuenta las diversas dimensiones de la variable educación religiosa católica, existe un alto índice de correlación con respecto a la formación en valores.

Cabe resaltar la diferencia en el valor global obtenido para la variable educación religiosa católica, que arroja un alto índice de correlación; mientras que algunas dimensiones de dicha variable, como la pastoral educativa y la formación familiar, presentan más bien un índice de correlación solamente medio o moderado. Para algunos estadísticos, esto puede deberse al factor de redondeo propio de la fórmula empleada, que arroja resultados ligeramente divergentes. Para otros, es un ejemplo típico de "el falso promedio", en donde la suma de promedios parciales no será nunca exactamente igual al promedio que arrojan los datos tomados unitariamente.

\section{CONCLUSIONES}

1. Existe una influencia significativa (índice de correlación alta) de la Educación Religiosa Católica en la formación en valores en los estudiantes del segundo grado de educación secundaria 2010 de la I.E. "San Jacinto", del distrito de Nepeña, lo que se evidencia en el 0.71189 de correlación positiva.

2. A pesar de la crisis en que está inmersa la sociedad y por encima de los problemas que específicamente experimentan los jóvenes estudiantes de la I.E. "San Jacinto", no pierden sus valores fundamentales, entre ellos el valor del respeto, que alcanzó el mayor porcentaje de puntaje acumulado, junto con los valores de responsabilidad y puntualidad. Sin embargo, los valores de participación y solidaridad, presentaron menores puntajes, lo cual permite concluir que hay una influencia de la cultura individualista en los estudiantes.

3. En el nivel de correlación entre la dimensión pastoral educativa y la variable formación en valores se evidencia un índice de correlación medio o moderado. Se puede deducir que las actividades desarrolladas en la pastoral educativa no habrían articulado a los diferentes agentes educativos, con ausencia de vinculación entre la plana docente, administrativa y padres de familia con las iniciativas del área en mención.

4. En el nivel de correlación entre la dimensión área de educación religiosa y la variable formación en valores, que mostraron un nivel de correlación medio o moderado, se concluye que podría faltar un manejo adecuado del área de 
educación religiosa, sobre todo por el papel fundamental que juega "lo religioso" en la vivencia interior de los jóvenes heredado de los padres y de la comunidad circundante.

5. La dimensión formación familiar presentó un índice de correlación alta con respecto a la variable formación en valores. Este nivel de correlación podría deberse, a pesar del crítico panorama familiar que experimentan los jóvenes, al escaso acompañamiento de sus familiares cercanos, o que han sabido asimilar el sacrificio y las enseñanzas que recibieron de sus padres.

\section{NOTAS}

1 Carta Pastoral de los Obispos del Perú. Caminemos con el Señor de la Historia, de la Vida, y de la Esperanza. Lima. 2001.

2 Documentos completos del Vaticano II, Gaudium et Spes, 61. Bilbao: Editorial Mensajero; 1984.

3 Gismondi M. Descontrol en adolescentes, Mar Adentro, diciembre 2011, Secc. Voces juveniles, pág. 8 , col. 1 .

4 Curibanco J. Alcoholismo: la lucha contra este mal comienza en casa, Mar Adentro, febrero 2012, Secc. Familia: comunidad de vida, pág. 9, col. 1.

5 GiSMONDi M. Bullying. Enfrentando al enemigo desde el hogar. Mar Adentro, julio 2012, Secc. Familia: comunidad de vida, pág. 5, col. 1.

6 GiSMONDi M. Suicidio adolescente y juvenil, demanda pronta atención, Mar Adentro, septiembre 2012, Secc. Familia: comunidad de vida, pág. 5, col. 1. 